\title{
Orthosurgical Management of Parry Romberg Syndrome: Long-Term Follow-Up of a Clinical Case
}

\author{
Abdoul Hafizou Rabe ${ }^{1, *}$, Faiçal Choumi ${ }^{2}$, Claude Garcia ${ }^{3}$, Yasmina EL Honsali ${ }^{1}$, Fatima Zaoui ${ }^{4}$, Nawal Bouyahyaoui ${ }^{4}$, and Loubna \\ Bahije $^{4}$ \\ ${ }^{1}$ Resident Dentist in Dentofacial Orthopedics, Mohammed V University in Rabat, Faculty of Dentistry-Rabat, avenue Mohammed El Jazouli, Madinat Al \\ Irfane, Rabat, Morocco \\ ${ }^{2}$ Associate Professor of Higher Education in Stomatology and Maxillofacial Surgery at the Moulay Ismail-Meknès Military Hospital, Faculty of Medicine and \\ Pharmacy of Fez, Sidi Mohamed Ben Abdallah University, Fez, Morocco \\ ${ }^{3}$ Specialist in Maxillofacial Surgery in Marseille-France \\ ${ }^{4}$ Professor of Higher Education in Dentofacial Orthopedics, Mohammed V University in Rabat, Faculty of Dentistry, Rabat, Morocco
}

*Corresponding author: Abdoul Hafizou Rabe, Resident Dentist in Dentofacial Orthopedics, Mohammed V University in Rabat, Faculty of Dentistry-Rabat, avenue Mohammed El Jazouli, PO box -6212, Madinat Al Irfane, Rabat, Morocco, Tel: 00212680962982; E-mail: abdoulhafizou_ rabeamani@um5.ac.ma

Received: 10 Dec, 2021 | Accepted: 22 Dec, 2021 | Published: 29 Dec, 2021

Citation: Rabe AH, Choumi F, Garcia C, Honsali YEL, Zaoui F, et al. (2021) Orthosurgical Management of Parry Romberg Syndrome: Long-Term FollowUp of a Clinical Case. Int J Dent Oral Health 8(1): dx.doi.org/10.16966/2378-7090.387

Copyright: (c) 2021 Rabe AH, et al. This is an open-access article distributed under the terms of the Creative Commons Attribution License, which permits unrestricted use, distribution, and reproduction in any medium, provided the original author and source are credited.

Abstract

Background: Progressive hemifacial atrophy is a rare disorder with skeletal and soft tissue changes in the affected half of the face.

Objectives: The main objective was to respond to the patient's aesthetic needs.

Methods: The authors report one rare case of a young female patient diagnosed with Parry-Romberg syndrome at eight years of age. Clinical examination of the patient revealed evident facial asymmetry, and atrophy of the other tissues on the left side of the face. The final diagnosis of Parry-Romberg syndrome was based on the clinical features. Orthodontic treatment at the age of 12 , followed by bimaxillary surgery with autogenous bone grafting and filling with fat injections at the age of 18 was suggested to the patient.

Results: These treatments provided satisfactory aesthetic, speech, and masticatory function results.

Conclusions: Orthodontic treatment followed by bone grafting and filling with fat injections could improve the facial aesthetic.

Keywords: Parry-Romberg syndrome; Oral manifestations; Reconstructive surgery

\section{Introduction}

The first published reports on progressive facial hemiatrophy were attributed to Parry in 1825 and Romberg in 1846 [1]. It is generally limited to half of the face but may affects the arm and trunk [2].

This syndrome is characterized by atrophy of the subcutaneous and adipose tissue of the hemiface. It is a rare disease with clinical and psychological sequelae, especially if appearing at a very young age [3-7].

Many authors have attempted to establish its various clinical manifestations, such as dermatological and ophthalmological, as well as its fairly frequent associations with neurological and endocrine diseases. However, very few authors have focused on its dentomaxillofacial manifestations and their management [2].

The most frequently cited oral manifestations are odontomas, root canal resorptions, delayed tooth eruptions, and the reduction in the size of the mandibular arch leading to functional disorders [2].
The objective of this article is to present the long-term follow-up of a patient with Parry Romberg syndrome at the early age of 3 years old, however having not consulted her orthodontist until the age of 8 , who then provided her with orthodontic care until the age of 18 years old before undergoing orthognathic surgery to correct the late growth anomalies of the maxillo-mandibular skeleton followed by fat injection and fillers to correct the facial asymmetry.

\section{Case Report}

\section{Patient concerns}

An 8-year-old child was referred, due to dental and maxillofacial issues. She was also complaining of her face's unsightly appearance. The patient had no familial history of specific disease or syndrome. Her 3 years old photo showed the first clinical signs (patches of skin depigmentation on the left hemiface with hair loss); her mother linked it up to a traumatic fall that occurred when she was 2 , and her right clavicle had even been fractured following that accident. There was no 
family history of a similar condition; she was not on any medication or known to have any chronic illness.

\section{Diagnostic aids}

In the initial extraoral examination, the facial asymmetry with heterogeneous skin hyperpigmentation, atrophy of the whole left hemiface area, more pronounced atrophy of the lips, noticeable when she's smiling and, and a "saber-cut" scar visible in the lower-left gonial area of the mandible was observed (Figure 1).

The intraoral examination showed a disturbed anterolateral occlusion on the left side which makes chewing very difficult; the left maxilla is narrower than the right one and the mandibular arch has a lyre shape. The left hemi-tongue and the soft palate are atrophied (Figure 1).

From a cephalometric point of view (Steiner and Tweed analyze), and Cone-beam computed tomography (Figure 2), the patient presented a class I hyper divergent skeletal pattern with the mandibular incisors protruded (Table 1) and the left maxilla narrowed.

Based on the clinical features, a diagnosis of Parry-Romberg syndrome was made. Routine blood investigations were carried out which revealed all values were normal.

\section{Treatment}

The patient followed an orthodontic treatment at the age of 12 to 16 , which made it possible to partially balance the occlusion in order to support the patient in her growth phase, to avoid a very severe asymmetry and to reach adulthood with the minimum of functional and aesthetic sequelae.

Difficulties were encountered because of the reduced bone support on the left side which complicated the control of mandibular molar torque at this level. Correction of the left lateral open bite was surgical, associated with lingual rehabilitation.
Only orthodontic treatment was not good enough for that case's management. We waited until the end of growth to avoid any recurrence related to the exacerbation of the atrophy during growth. Thus, an orthodontic retainer fixed in the mandible and removable in the maxilla was kept in place for 2 years.

At the end of growth, despite the well-followed orthodontic treatment, the facial asymmetry was evident and the occlusion plane was oblique from bottom to top towards the left side, orthosurgical management was required to correct the skeletal and occlusal deformities.

At the age of 18 , the patient received a 6 -month pre-surgical orthodontic preparation and subsequently underwent an orthognathic surgery, and lipostructure of the face (Figure 3). A Lefort I osteotomy with an intermaxillary disjunction to advance the maxilla and lower the left maxilla, with the placement of iliac bone graft on the left side, which made it possible to correct the open bite lateral and have a horizontal occlusal plane. A bilateral sagittal split osteotomy of the mandible (BSSO) with mandibular recoil, derotation, recentering, and an injection of fat in the atrophied areas of the labial and left cheekbones were also realized.

\section{Outcomes}

The post-surgical cephalometric assessment (Table 1) showed an improvement in the aesthetics of the patient who went from hyperdivergence to normodivergence skeletal pattern with a normal angle of the mandibular incisors.

At the age of 20, a second surgical intervention was performed to remove the osteosynthesis mini-plates following an infection in the left jaw. At the same time, the patient received fat injections in the upper lip and left cheek and the injection of fillers in the forehead to improve his facial aesthetics (Figure 4).
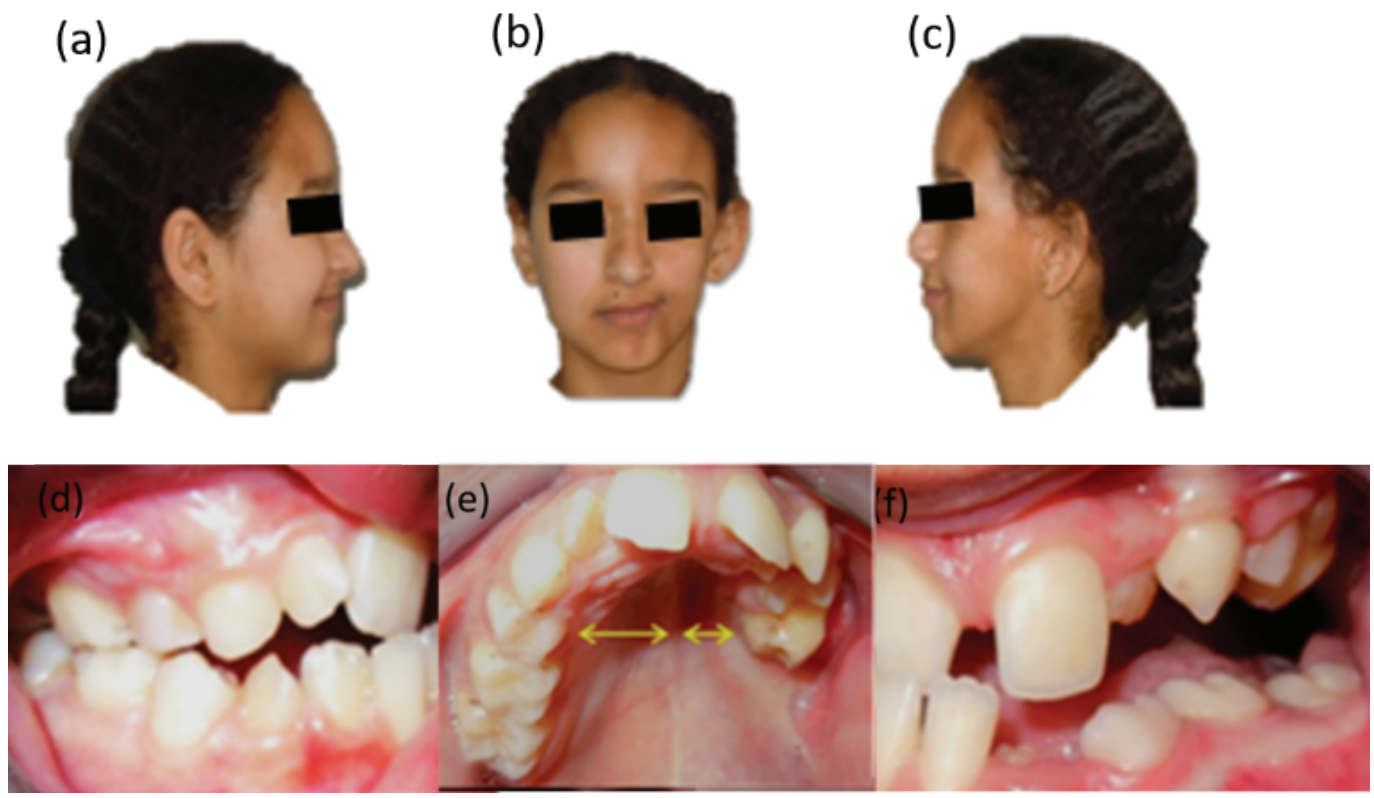

Figure 1: $(a, b, c)$ Extraoral view of prominent left hemifacial atrophy before iliac bone grafts and injection of fat: (a) right lateral view, (b) frontal view, and (c) left lateral view. (d, e, f) Intraoral view of occlusion disturbed on the left side: (d) right lateral view, (e) frontal view, and (f) left lateral view. 


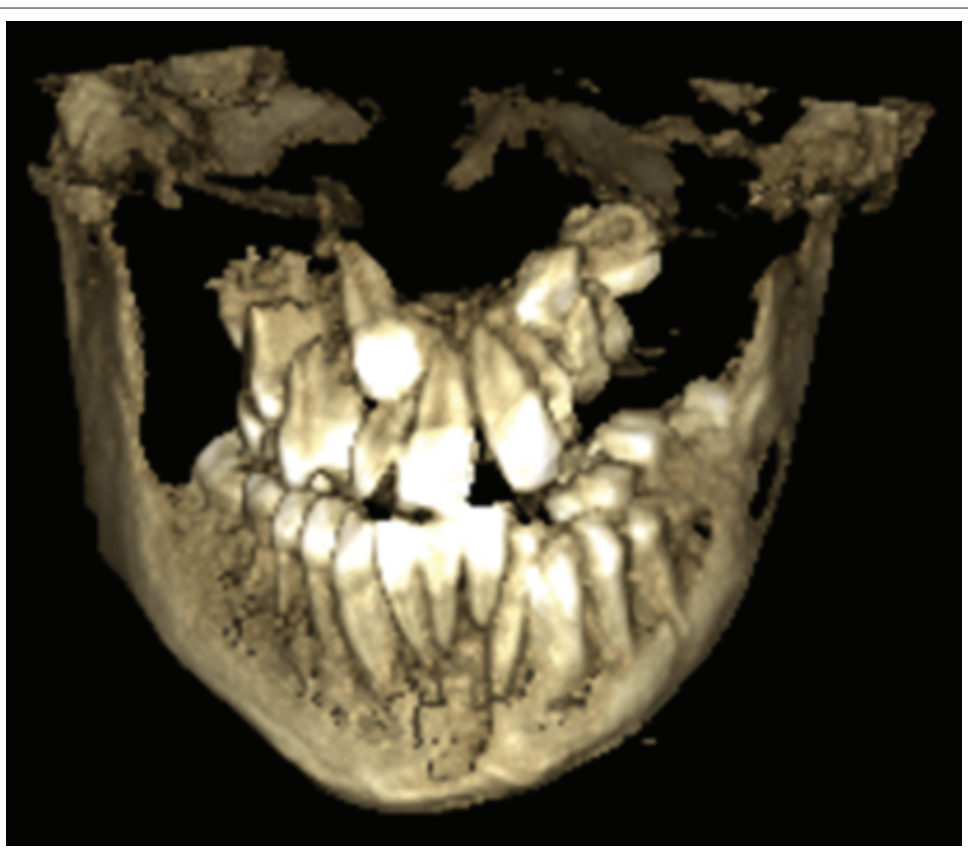

Figure 2: Cone-beam computed tomography showing the left maxilla narrowed.

(a)

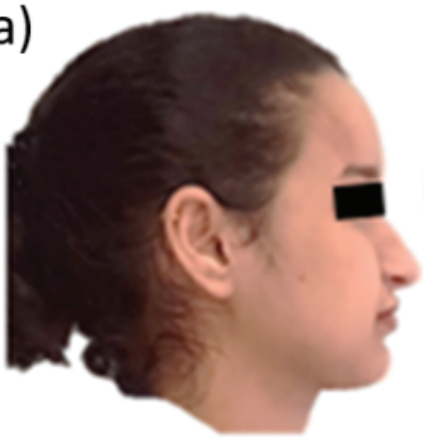

(b)

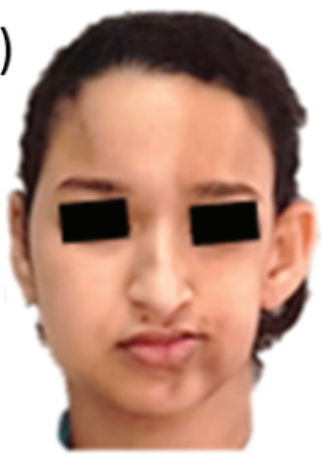

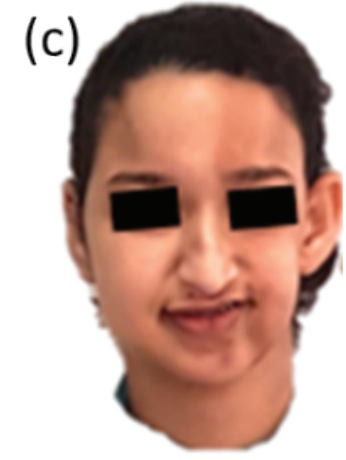

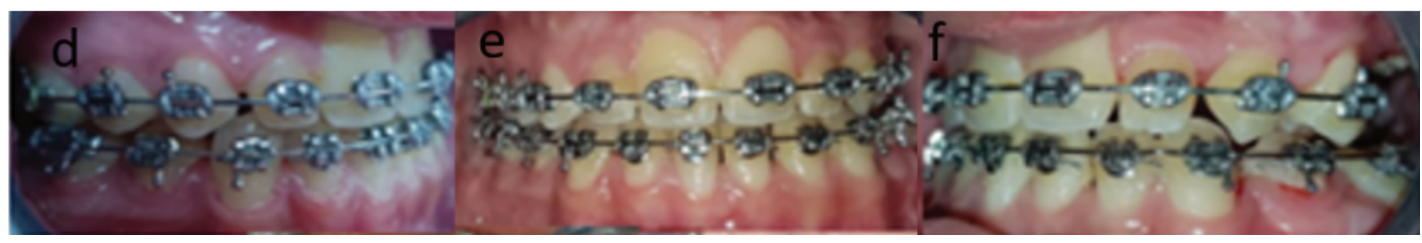

Figure 3: $(a, b, c)$ Extraoral view of left hemifacial atrophy correction after iliac bone grafts and injection of fat: (a) right lateral view, (b) frontal view, and (c) left lateral view. (d, e, f) Intraoral view of occlusion correction on the left side: (d) right lateral view, (e) frontal view, and (f) left lateral view.

\section{Follow-up}

In 2020, after a 12-year follow-up, the patient presented a stable result (Figure 3). She is satisfied with the augmentation volume and contours of the face, and the resolution of sclerosis which led to regaining selfconfidence. In perspective, a maxillary bone graft followed by a filling injection to have more aesthetic results were planned.

\section{Discussion}

An orthopedic and orthodontic treatment on a patient presenting Parry Romberg syndrome is aimed at correcting facial asymmetry.
The spontaneous progression without any treatment during the craniofacial growth phase will lead to worsening of the maxillary and mandibular asymmetry, especially the ramus and the longer condyle on the healthy front compared to the other one $[8,9]$.

The aim of the surgical treatment is to obtain a class I dental relationship with a favorable muscular balance and to reduce facial atrophy by injection of fat and filling products. It is recommended to wait for the cessation of the atrophic process before deciding on a restorative intervention, for fear of a possible flare-up of the disease and to have stable and lasting results. 
Table 1: Initial and after cephalometric assessment.

\begin{tabular}{|l|c|c|c|}
\hline \multicolumn{2}{|c|}{} & $\mathbf{5 / 2 0 0 6}$ & $\mathbf{7 / 2 0 0 8}$ \\
\hline FMIA & $67^{\circ} \pm 3$ & $53^{\circ}$ & $61^{\circ}$ \\
\hline IMPA & $25^{\circ} \pm 3$ & $31^{\circ}$ & $28^{\circ}$ \\
\hline SNA & $88^{\circ} \pm 3$ & $96^{\circ}$ & $91^{\circ}$ \\
\hline SNB & $82^{\circ} \pm 2$ & $78^{\circ}$ & $78^{\circ}$ \\
\hline ANB & $80^{\circ} \pm 2$ & $74^{\circ}$ & $74^{\circ}$ \\
\hline AoBo & $2^{\circ} \pm 2$ & $4^{\circ}$ & $4^{\circ}$ \\
\hline Plan of occ & $0 \mathrm{~mm} \pm 2$ & $1 \mathrm{~mm}$ & $1 \mathrm{~mm}$ \\
\hline Angle Z & $10^{\circ}$ & $1^{\circ}$ & $10^{\circ}$ \\
\hline Upper lip & $75^{\circ} \pm 5$ & $64^{\circ}$ & $77^{\circ}$ \\
\hline Total chin & $/$ & $8 \mathrm{~mm}$ & $11 \mathrm{~mm}$ \\
\hline Ht Facial Post & $/$ & 14 & 11 \\
\hline Ht facial Ant & $65 \mathrm{~mm}$ & 40 & 40 \\
\hline Post Ant Index & 0.69 & 62 & 62 \\
\hline
\end{tabular}

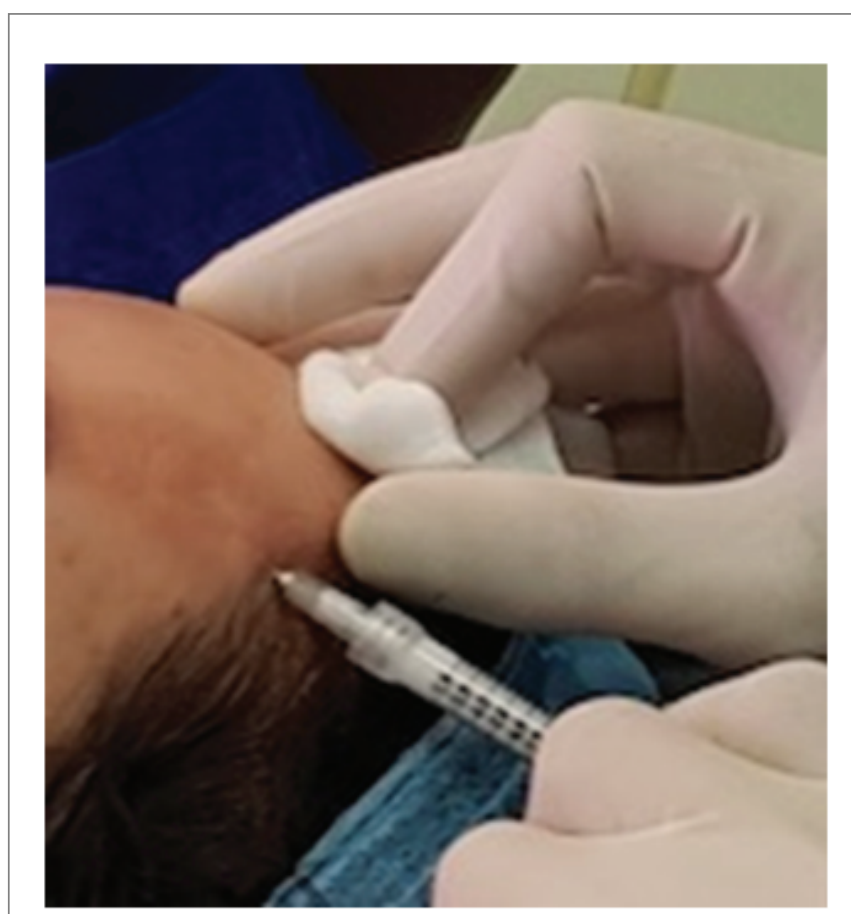

Figure 4: Injection of fillers in the left forehead.

The surgical treatment has two components:

- Orthognathic surgery: a bimaxillary osteotomy to obtain a skeletal class I dental relationship. A Lefort I maxillary osteotomy can be associated with intermaxillary disjunction; and if the bone displacements are important one can use autologous bone grafts most often of iliac origin, as in the case of our patient. A bilateral sagittal split osteotomy of the mandible (BSSO) of DALPONT-OBWEGESER allows the possibility of derotation and mandibular recentering.
- Lipofilling: For moderate or localized atrophy by the injections of adipocytes and/or a de-epidermized skin flap can be used. For large atrophies, the free parascapular flap can be used $[10,11]$.

\section{Conclusion}

Orthodontic treatment followed by bone grafting and filling with fat injections can be done to improve the facial aesthetic. After the Ortho-surgical management, the prosthetic phase completes the dental occlusion on the affected side.

\section{Patient Consent}

Written consent was obtained for publication.

\section{Financial Support and Sponsorship}

Nil.

\section{Conflicts of Interest}

No conflicts of interest.

\section{References}

1. Zafarulla MY (1985) Progressive hemifacial atrophy: a case report. Br J Ophthalmol 69: 545-547.

2. Fea AM, Aragno V, Briamonte C, Franzone M, Putignano D, et al. (2015) Parry Romberg syndrome with a wide range of ocular manifestations: a case report. BMC Ophthalmol 15: 119.

3. Tolkachjov SN, Patel NG, Tollefson MM (2015) Progressive hemifacial atrophy: a review. Orphanet J Rare Dis 10: 39.

4. Khamaganova I (2018) Progressive Hemifacial Atrophy and Linear Scleroderma En Coup de Sabre: A Spectrum of the Same Disease? Front Med (Lausanne) 4: 258.

5. Al-Aizari NA, Azzeghaiby SN, Al-Shamiri HM, Darwish S, Tarakji B (2015) Oral manifestations of Parry-Romberg syndrome: A review of literature. Avicenna J Med 5: 25-28.

6. Miller MT, Spencer MA (1995) Progressive hemifacial atrophy. A natural history study. Trans Am Ophthalmol Soc 93: 203-217.

7. Abdelnour JGW, Abdelnour YGW, Kerollos RAB, Mahmoud ZIT (2019) Parry-Romberg syndrome associated with en coup de sabre in a patient from South Sudan-a rare entity from East Africa: a case report. J Med Case Rep 13: 138.

8. McNamara JA Jr (1973) Neuromuscular and skeletal adaptations to altered function in the orofacial region. Am J Orthod 64: 578-606.

9. Enlow DH, Kuroda T, Lewis AB (1971) Intrinsic craniofacial compensations. Angle Orthod 41: 271-285.

10. Onesti MG, Monarca C, Rizzo MI, Mazzocchi M, Scuderi N (2009) Minimally invasive combined treatment for Parry-Romberg syndrome. Aesthetic Plast Surg 33: 452-456.

11. Qiao J, Gui L, Fu X, Niu F, Liu J, et al. (2017) A Novel Method of Mild to Moderate Parry-Romberg Syndrome Reconstruction: ComputerAssisted Surgery With Mandibular Outer Cortex and Fat Grafting. J Craniofac Surg 28: 359-365. 